\title{
Contemporary Adult Orthodontics with Aligners-New Mindset Needed
}

\author{
Wolfgang Richter* \\ Mannedorf, Zurich, Switzerland
}

*Corresponding author: Wolfgang Richter, Professor, Weingartenstrasse 8 CH-8708, Maennedorf, Zurich, Switzerland, E-mail: wrichterdr@aol.com

Received: 07 Nov, 2020 | Accepted: 25 Nov, 2020 | Published: 01 Dec, 2020

Citation: Richter W (2020) Contemporary Adult Orthodontics with Aligners-New Mindset Needed. Int J Dent Oral Health 7(1): dx.doi. org/10.16966/2378-7090.345

Copyright: (C) 2020 Richter W. This is an open-access article distributed under the terms of the Creative Commons Attribution License, which permits unrestricted use, distribution, and reproduction in any medium, provided the original author and source are credited.

According to current estimates, almost half of all orthodontic treatments for adults are now performed with aligners. At the same time, technological development has developed exponentially due to digitalization and has opened up completely new treatment options for us, dentists.

However, numerous patients with hopeless dentition conditions also arrived, hoping for a miracle from aligners. Since all these people were initially referred to a general dentist, aligners have probably indirectly contributed to saving a considerable number of teeth and increased the level of dental awareness in the population.

It is, of course, a question of a correct indication of whether treatment with aligners is superior, equal, or inferior to other forms of therapy. The dentist who places implants has, certainly, attended appropriate training courses, as has the dentist who carries out aligner therapy on his patients.

Aligner shops in which no dentists work, where the splints are sent out and no follow-ups are carried out, are not acceptable. It is also unacceptable if it is demanded that only orthodontists are allowed to carry out the aligner treatment ("Aligner methods are only recommended for a well-trained dentist who is experienced in the use of the entire orthodontic range of diagnostics and can perform the entire therapeutic spectrum.").

In the recent Quintessence Webinar (Updates on Aligner Orthodontics) on October 8, 2020, Dr. Sandra Tai answered the question of the moderator Prof. Dr. Ralf Radlanski that in large parts of the world there is no specialist dentist for orthodontics at all and that such a restriction would not allow millions of people to receive treatment.

Invisalign alone has produced more than 200 million splints up to date and over 4 million patient cases have been completed. Over 100,000 dentists have been trained at courses, only a small proportion of who are specialists in orthodontics. If you add the other clear aligner systems up, you immediately realize that orthodontists only just wouldn't be able to meet the demands.
Cloud-based treatment planning, visualization workflow with a personalized view of the planned treatment result and digital documentation of the therapy are changing orthodontics incredibly fast. Especially when talking to older or less digitally affine colleagues, I often find that it is an enormous challenge and therefore stressful to recognize and accept new digital possibilities. A new mindset is needed.

In some countries, especially in Europe, this is a minefield of professional politics. In German-speaking countries, there is a passionate dispute about who is allowed to perform the intraoral scan. One gets lost in details, whether it is a photo status or a manipulation in the mouth.

Following the basic rules of delegation in medical activities would be quite simple. The delegation of medical tasks is possible, in principle, but it must not be done in a generalized manner but must take the following aspects into account individually:

-Condition of the patient

-Profession-specific qualification of the non-medical staff

-The instruction, coordination and success control as well as their documentation is entirely the responsibility of the dentist.

Delegation of duties to auxiliaries is part of practice in many countries already. But on the other hand, it isn't clear in a few countries which duties in detail are allowed to be performed by auxiliaries.

This procedure is also common for all Artificial Intelligence (AI) applications in dentistry (and of course in medicine as well), especially in image recognition.

The teaching of specialist knowledge for intraoral scanning can be done by the dentist himself or herself, or you can book a course for your dental assistants (i.e. Straumann (Implant Manufacturer, Basel, Switzerland) is offering a training seminar especially for dental assistants).

Finally, due to the new digital possibilities, especially in the treatment of adults, the question is allowed, which diagnostic findings 
can be expected in the adult jaw with dentoalveolar movements (not skeletal) by the preparation of a cephalometric X-ray.

Of course, a comprehensive assessment with documentation of the entire facial and jaw situation must be carried out before an alignment therapy. This is easily possible with panoramic X-ray in terms of apical and periapical brightening, retained roots or teeth, and horizontal and vertical bone loss.

In adjunctive orthodontics, like aligner therapy, for anterior teeth in adults, a cephalometric X-ray is dispensible whereas it's the gold standard in skeletal changes in adolescents.

The superimposition, made possible by numerous software tools, allows for planning and control (including the inclination of the anterior teeth).

Orthodontic and dental measurements must be carried out legeartis regardless of the size and scope of the treatment. However, this term is in flux due to the rapid development of the possibilities in diagnostics and treatment.
Updated guidelines are systematically developed aids for dentists to make decisions in specific diagnostic and therapeutic situations. They should reflect current scientific knowledge and best practices in practice and provide more security in aligner Orthodontics.

These guidelines should include:

- Rationale for aligner treatment for general Dentists;

- Optional delegation of duties to auxiliaries;

-Referral guidelines to Orthodontists;

-Specifics of case complexity and treatment considerations.

I would like to kindly request guidelines from Professional Societies and Universities soon, which take into account the further development of aligner orthodontics so that we can help as many patients as possible to achieve a beautiful smile and better dental health. 